\title{
Slowing the decline
}

\section{The search is on for disease-modifying treatments for Parkinson's disease, but, as Ruth Williams discovers, developing a compound is only part of the problem.}

P arkinson's disease is characterized by progressive loss of dopaminergic neurons in the nigrostriatal region of the brain. To compensate, patients commonly receive the dopamine precursor levodopa (L-DOPA) or, less commonly, dopamine agonists such as pramipexole. These relieve symptoms, but deep in the brain, the disease rages on. More neurons are lost, symptoms worsen, complications arise and quality of life dwindles. These treatments also come with an array of unpleasant side effects. Thus, for the past two decades, researchers have been hunting for drugs that slow, stop or, better still, reverse the disease pathology.

Such disease-modification strategies have shown success in autoimmune disorders including multiple sclerosis and rheumatoid arthritis; however, the pathological process of Parkinson's disease is proving to be a tougher beast to tame.

There is hope. Neurologists suspect that some new and existing Parkinson's disease drugs have disease-modifying qualities. "The most promising is probably rasagiline," says Warren Olanow of the Mount Sinai School of Medicine in New York. "My highest level of enthusiasm is still for trophic factors," says Eugene Johnson of the Washington University School of Medicine. M. Flint Beal of Weill Cornell Medical College in New York, by contrast, has high hopes for coenzyme Q10 - widely available as a nutritional supplement (see sidebar, page S10).

This lack of consensus among researchers in the field might be contributing to the difficulties in finding a suitable drug. "If you're going to come up with some meaningful finding, it has to be something that the general Parkinson's disease community would accept as valid," says Eric Ahlskog, a neurologist at the Mayo Clinic in Rochester. He adds, "all of these studies that have been assessments of progression seemingly raise more questions than they answer."

\section{Troublesome trials}

Take coenzyme Q10. There is good reason to think that boosting its levels might be neuroprotective in Parkinson's disease as it is a normal component of the electron-transport chain in mitochondria and dysfunctional mitochondria are believed to contribute to Parkinson's disease pathology (see page S2). It has also been shown to be low in the serum, spinal fluid and brain cortex of Parkinson's disease patients. Boosting coenzyme Q10 might therefore boost mitochondrial function and save neurons. Indeed, it has been shown to be neuroprotective in vitro and in animal models of Parkinson's disease $^{1,2}$. Clinical trials, however, have given mixed results.

A small phase II trial (QE2) supported by the National Institute for Neurological Disorders and Stroke (NINDS) showed that 20 patients taking 1,200 milligrams per day of coenzyme Q10 for 12 months had a $44 \%$ slower degeneration rate than those on placebo ${ }^{3}$. Degeneration is assessed by change in the Unified Parkinson's Disease Rating Scale (UPDRS) - a set of scores derived from interviews and clinical observations that assess a patient's motor skills, behaviour and ability to perform daily tasks.

Despite these promising results however, a subsequent NINDS-funded coenzyme Q10 trial showed no difference between treatment and placebo arms - both in fact degenerated more slowly than usual ${ }^{4}$. "It was a curious result for which there is no really good explanation," says Beal. He suggests that differences in practice and trial design might be to blame. "[At the time of the second NINDS trial] patients were being put on Sinemet [a drug containing L-DOPA] earlier than previously, and the duration of the trial was shorter, giving it less of a chance to show a difference," he says.

The case for neurotrophic factors as disease modifiers also began with sound scientific rationale and promising laboratory results, but again translated into complicated results in clinical trials. No benefits were seen when glial-derived

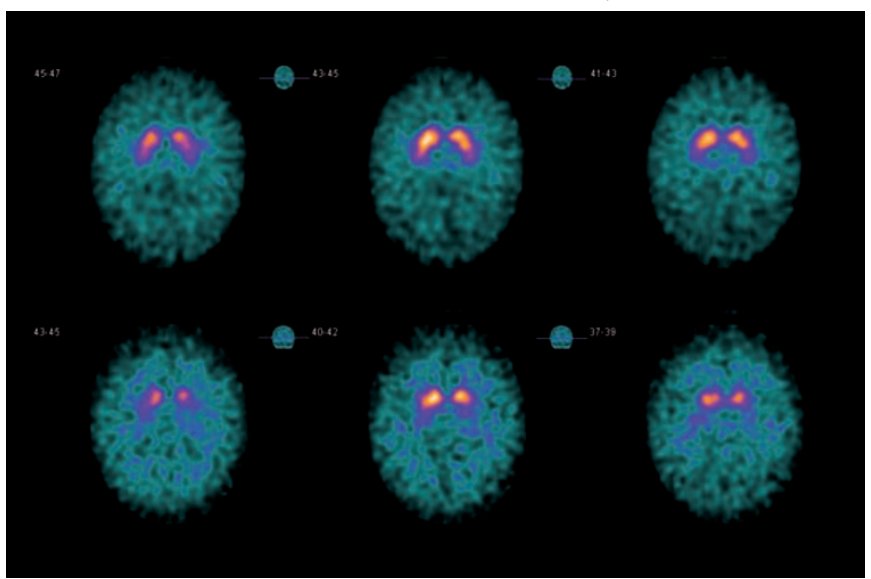

Single photon-emission computed tomography (SPECT) images show loss of dopamine transporter binding in Parkinson's disease (bottom) compared with a normal pattern of uptake (top).

neurotrophic factor was delivered by catheter into patients' brains ${ }^{5,6}$, and phase I and II trials of gene therapy with neurturin were mildly encouraging but inconsistent ${ }^{7}$ (see page S15).

Symptomatic effects can also confuse trial results, as shown by the first diseasemodification drug trial, the Deprenyl and Tocopherol Antioxidative Therapy of Parkinsonism (DATATOP) study, which involved selegiline (L-deprenyl).

Selegiline is a monoamine oxidase inhibitor and is thought both to slow the degradation of dopamine and to reduce oxidative stress inside neurons. This latter activity was considered a possible means to curb neurodegeneration - hence the DATATOP trial. In the trial, selegiline was given to patients early in disease, before they needed to take L-DOPA. These patients could last nine months longer than placebo-taking patients before starting L-DOPA treatment - which was interpreted as evidence of selegiline's neuroprotective effect. This conclusion was later questioned, however. Critics suggested that selegiline's mild symptomatic benefit (from slowing dopamine degradation and therefore enhancing endogenous dopamine levels) had not been adequately taken into account in the trial design ${ }^{8}$.

This symptomatic issue also dogs trials of creatine - another mitochondrial activityboosting nutritional supplement. Creatine has been shown to be neuroprotective in vitro and in animal models of Parkinson's disease, and has shown promising results in a phase II NINDS-sponsored study9. Ahlskog points out, however, "If you look at the curves, the group that was started on creatine compared to placebo [shows] a small symptomatic effect." 
Olanow concurs, "Creatine in particular is readily confounded by its potential effect on muscle that could make a patient feel better and therefore potentially interfere with the UPDRS score."

\section{Tweaking trial design}

As researchers must pick out true diseasemodifying effects from any symptom-relief noise, trial design is key. Indeed, poor trial design was the undoing of the Earlier vs Later L-DOPA (ELLDOPA) trial. Patients were given L-DOPA for 40 weeks, early in disease. They spent a further two weeks drug-free to see whether their baseline UPDRS score was better than patients who had not taken L-DOPA. A mild neuroprotective effect was observed, but was later disregarded when the two-week wash-out period was criticized for being too short ${ }^{10}$.

A newer trial design that avoids the need for a wash-out period is the so-called delayed start. This involves giving one-half of the subjects the test drug for the entire study period, while the remainder receive placebo for the first half followed by the test drug for the second half. "If the early treatment group continues to do better than the delayed treatment group then it argues that you have done something that influences the underlying disease process," explains Olanow. If the effect was purely symptomatic, the two groups should be indistinguishable.

The recent Pramipexole on Underlying Disease (PROUD) trial employed the delayed start to test the suspected disease-modifying effects of the dopamine agonist pramipexole. It was found to have none ${ }^{11}$. The TVP-1012 in Early Monotherapy for Parkinson's Disease Outpatients (TEMPO) and Attenuation

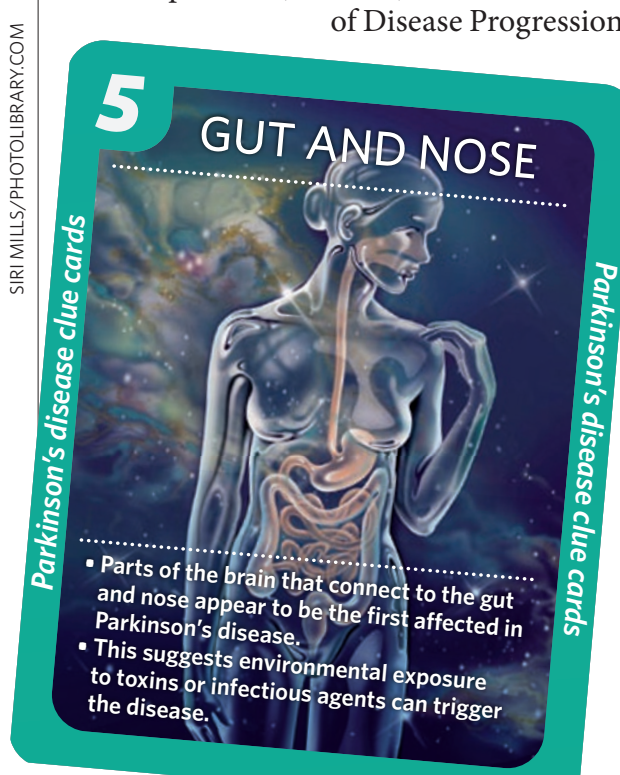

with Azilect Given Once-daily (ADAGIO) trials used the delayed-start method to test rasagiline - similar to selegiline but with less harmful metabolites ${ }^{12,13}$. Results from both supported the disease-modifying potential of rasagiline, although there were some oddities. In the TEMPO trial, results consistent with disease modification were seen with two milligrams per day of rasagiline; however, in the ADAGIO trial, disease-modifying effects were seen only with one milligram. "We're still trying to determine why that should have occurred," says Olanow. He suggests that in the ADAGIO trial, the higher dose created a symptomatic effect that overshadowed any disease-modification effect - "a floor effect". This was not seen in the TEMPO trial because the subjects had more advanced disease; a yet higher dose of rasagiline might have created a floor effect.

Although many believe rasagiline is a promising disease-modifying drug, the lack of reproducible results is a concern.

\section{The need for better tools}

Ahlskog suggests that the lack of reproducibility is largely the fault of the measuring tool. The UPDRS, he explains, was designed to assess the benefit of symptomatic treatments for Parkinson's disease. "With these you see big changes on the scale," he says. However, he points out, "this is not a real robust rating scale for distinguishing small differences that occur over a relatively short period of time, such as one year." On top of that, Parkinson's disease symptoms can be relatively changeable, says William Marks, a specialist in movement disorders at the University of California, San Francisco. "We've got a variable disease," he says, and, "we've got imperfect, subjective tools."

"The holy grail," says Kenneth Marek, president and senior scientist at the Institute for Neurodegenerative Disorders in New Haven, "is to identify markers of disease progression." Indeed, agrees Olanow, "Imaging and other biomarkers are desperately needed." On this issue, the entire Parkinson's disease research community appears to be united.

Current imaging approaches for Parkinson's disease lack the necessary sensitivity for detecting small changes (see page S11). In a Parkinson's disease patient, you might expect to see a reduction in the number of dopaminergic neurons of about 5 to $10 \%$ per year, says Marek, but most of the drugs being tested are expected to slow neuronal loss by around 25 to $30 \%$, "So, instead of a $5 \%$ loss you might get $3.5 \%$ - that would be an important change for the patient, but it's a very hard change to detect," he explains.

Nevertheless, even without a sensitive validated biomarker, trials of disease-modifying

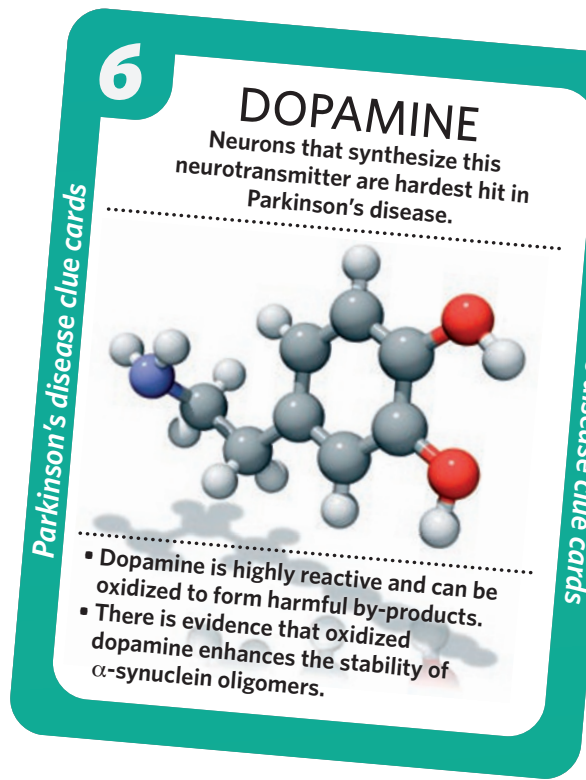

treatments are underway. Coenzyme

Q10 and creatine are in phase III trials, a phase I/II study has started for neurturin gene therapy and a longer-term trial is underway for rasagiline. The desire to find a diseasemodifying therapy is understandably strong. But as neither the UPDRS nor current imaging tools are considered adequately sensitive, and since there is unanimous agreement that biomarkers are required, Ahlskog asks, "are we prematurely doing these trials?"

Rasagiline's full potential, for example, might become clear only over years of treatment. These extensive clinical trials are expensive, and some researchers argue that funds would be better spent on basic research into Parkinson's disease. "I would personally be inclined to spend more resources [on] looking for the cause," says Ahlskog.

Nonetheless, if one of these drugs does yield consistent results, even with imperfect tools, we can be sure that its effect is real, which would be a huge boon to the millions of Parkinson's disease patients for whom symptomatic treatment can do only so much.

Ruth Williams is a freelance writer in London, United Kingdom.

1. Moon, Y. et al. J. Neurochem. 93, 1199-1208 (2005)

2. Beal, M. F. et al. Brain Res. 783, 109-114 (1998).

3. Shults, C. W. et al. Arch. Neurol. 59, 1541-1550 (2002)

4. NINDS NET-PD Investigators. Neurology $68,20-28$

(2007).

5. Nutt, J. G. et al. Neurology 60, 69-73 (2003)

6. Lang, A. E. et al. Ann. Neurol. 59, 459-466 (2006).

7. Marks, W. J. Jr et al. Lancet Neurol. 7, 400-408 (2008).

8. Ward, C. D. J. Neurol. Neurosurg. Psychiatry 57, 217-220 (1994).

9. NINDS NET-PD Investigators. Neurology $66,664-671$ (2006).

10. Fahn, S. J. Neural. Transm. 70 (suppl.), 419-426 (2006).

11. Schapira, A. et al. Parkinsonism Relat. Disord. 15 (suppl. 2), abstr. S81 (2009).

12: Parkinson Study Group. Arch. Neurol. 61, 561-566 (2004) 13. Olanow, C. W. et al. N. Engl. J. Med. 361, 1268-1278 (2009). 